\title{
Complexity and Fragility in Stability for Linear Systems
}

\author{
Aaron D. Ames and John C. Doyle
}

\begin{abstract}
This paper presents a formal axiomization of the notion that (proof) complexity implies (property) fragility and illustrates this framework in the context of the stability of both discrete-time and continuous-time linear systems.
\end{abstract}

\section{INTRODUCTION}

Understanding the robustness of systems with respect to a certain property plays a fundamental role in design and analysis; when systems are robust they tend to behave properly in both foreseen and unforseen circumstances. It is, moreover, often the case that the lack of robustness, or fragility, of a system is indicated when trying to prove that the system has a certain property. That is, that the complexity of a proof relates directly to-and in fact implies-the fragility of the property being proven. Yet this connection has, for the most part, remained anecdotal.

Taking the idea that complexity implies fragility beyond the anecdotal level has important practical consequences. On a general level, when using automated proof techniques to verify properties, e.g., stability of dynamical systems, it would be very useful to know that when such algorithms are producing longer and longer "proofs," or are unable to terminate altogether, that it is indicative of certain fragilities in the system being considered. On a more concrete level, it is typically easier to check that a proof is becoming more complex as opposed to verifying that a system is fragile with respect to a certain property; in the former case, a proof is already being constructed in order to prove the desired property. Therefore, establishing that complexity implies fragility in a formal and general context will have far-reaching ramifications.

The goal of this paper is to take the first steps toward providing a formal framework in which to understand how complexity affects fragility. In order to do so, it is necessary to first formalize the notation of property, proof and complexity, which is done through specifications intended to capture each of these notions. In particular, we introduce property specifications which consist of the set of problems being considered and the subset of properties displaying the desired property (this allows one to define the fragility of a problem), proof specifications which view proofs as certificates that are verified functionally, and (proof) complexity specifications which are functions from the set of proofs to the real numbers satisfying the property that as the complexity diverges, so must the fragility. Combining a property specification, proof specification and complexity specification yields a proofproperty-complexity (PPC) specification. In this framework,

A. D. Ames and J. C. Doyle are with the Control and Dynamical Systems Department, California Institute of Technology, Pasadena, CA 91125 \{ames, doyle\}@cds. caltech.edu we say that complexity implies fragility for a given PPC specification if the complexity of a proof that a problem has a property lower bounds the fragility of that problem.

To illustrate our framework for complexity and fragility, we begin by considering the stability of discrete-time linear systems. In this case, the property specification consists of linear systems and the subset of stable systems, the proof specification consists of the set of Hermitian positive definite matrices which are verified as proofs of stability through the discrete-time Lyapunov equation and the complexity specification is a function of the norm of the proof. Using the results presented in [4] and [16], we note that complexity implies fragility for this PPC specification; the simplicity of this result illustrates the concepts involved in a transparent manner.

Having motivated our framework for complexity and fragility in the context of discrete-time linear systems, we proceed to present a complexity implies fragility result in the context of the stability of continuous-time linear systems. In this case, the property specification again consists of linear systems and the subset of stable systems and the proof specification consists of the set of Hermitian positive definite matrices which are verified as proofs of stability through the continuous-time Lyapunov equation. Unlike the discretetime case, we adopt a somewhat unorthodox complexity specification related to the reciprocal of the norm of the inverse of a proof. Through the techniques outlined in [1], we are able to prove that complexity implies fragility for the PPC specification; as far as the authors are aware, this is a novel result.

The ideas introduced in this paper have drawn their inspiration from a wide variety of sources. For example, the results in [15] aided in the present formulation of complexity implies fragility, and [1] and [8] contributed to the view of fragility taken in this paper. The literature studying the fragility and sensitivity of linear systems in both discretetime (cf. [4], [12] and [16]) and continuous-time (cf. [2], [5], [6], [7], [10], [11], [14] and [17]) also has played a central role in providing a coherent and meaningful formulation of complexity and fragility in the context of linear systems. The exact way in which the aforementioned work has influenced this work will be made clear throughout the rest of the paper.

\section{Complexity And Fragility: The AXioms}

This section introduces an axiomatic approach to the notion of proof complexity and property fragility. These axioms are not meant to be all-encompassing, but rather lay the groundwork for the development of a systematic approach to understanding complexity and fragility. 
We refer the reader to the next section for a detailed example illustrating the definitions presented in this section.

Property Specification. Given a set of problems in the form of a metric space, a property is specified through the use of a subset of the problems that display this property. In particular, there is the following:

Definition 2.1: A property specification is a tuple:

$$
\left(\mathcal{X}, d_{\mathcal{X}}, \mathcal{G}\right)
$$

where

- $\left(\mathcal{X}, d_{\mathcal{X}}\right)$ is a metric space, consisting of the problems of interest,

- $\mathcal{G} \subset \mathcal{X}$ is the set of problems that display a specific property.

Note that $\mathcal{B}:=\mathcal{G}^{c}=\mathcal{X} \backslash \mathcal{G}$ is the set of problems that do not display a specific property.

Fragility. With the notion of a property specification in hand, given a problem displaying the property, i.e., an element of $\mathcal{G}$, one can define how "fragile" that problem is, i.e., how far the property is from not being true.

Definition 2.2: Given a property specification $\left(\mathcal{X}, d_{\mathcal{X}}, \mathcal{G}\right)$, the fragility of a problem $x \in \mathcal{G} \subset \mathcal{X}$ is given by:

$$
F(x):=\frac{1}{d_{\mathcal{X}}(x, \mathcal{B})}
$$

where $d(x, \mathcal{B})=\inf _{y \in \mathcal{B}} d_{\mathcal{X}}(x, y)$. Note that we have thus defined a function $F: \mathcal{G} \rightarrow \mathbb{R}_{+}=(0, \infty)$.

Remark 1: The quantity $d(x, \mathcal{B})$, and more generally property specifications (although they were not called such), has been studied in a variety of situations. For example, [8] considers numerous property specifications related to the matrix nearness problems, [1] studies $d(x, \mathcal{B})$ in the context of functions that play the role of "condition numbers" and [15] considers $d(x, \mathcal{B})$ in the context of linear programming.

Proof specification. Having established how to specify properties through the use of subsets, we present an axiomatic notion of "proofs" or "certificates" that can be "verified" functionally.

Definition 2.3: A proof specification is a tuple:

$$
(\mathcal{P}, \mathcal{R}, V)
$$

where

- $\mathcal{P}$ is a set, consisting of proofs or certificates,

- $\mathcal{R}$ is a vector space, termed the verification space,

- $V: \mathcal{X} \times \mathcal{P} \rightarrow \mathcal{R}$ is a function, termed the verification function, which must satisfy the property:

$$
V(x, p)=0 \quad \Rightarrow \quad x \in \mathcal{G} .
$$

Remark 2: It is certainly possible to consider more elaborate verification functions or collections thereof. For example, one could require that $\mathcal{R}$ is a partially ordered vector space (with the partial ordering denoted by $\geq$ ) and that there exists a collection of verification functions $V_{i}: \mathcal{X} \times \mathcal{P} \rightarrow \mathcal{R}$, $i \in\{0,1, \ldots, m\}$, satisfying the property:

$$
V_{i}(x, p) \geq 0 \quad \forall \quad i \in\{0,1, \ldots, m\} \quad \Rightarrow \quad x \in \mathcal{G} .
$$

The motivation for opting for a single verification function as given in Definition 2.3 is simplicity of exposition. Of course, in settings different than the one considered in the rest of this paper-the stability of linear systems - it may be desirable to utilize the more elaborate notion of verification functions. For example, in the context of proofs produced by sum-of-squares techniques [13], is seems likely that inequalities would be desirable. Similarly, when considering linear programs [15], inequalities also would be desirable.

Complexity specification. With the notions of property specification and proof specification in hand, we are able to define "proof complexity" or just "complexity." This will serve to tie together property and proof specifications, while allowing for the formalization of the notion that "complexity implies fragility."

Definition 2.4: Let $\left(\mathcal{X}, d_{\mathcal{X}}, \mathcal{G}\right)$ be a property specification and $(\mathcal{P}, \mathcal{R}, V)$ be a proof specification. A proof complexity specification or just complexity specification is a function:

$$
C: \mathcal{P} \rightarrow \mathbb{R}_{+}
$$

satisfying the property that for any pair of sequences $\left\{x_{i}\right\}_{i=0}^{\infty}$ and $\left\{p_{i}\right\}_{i=0}^{\infty}$ with $x_{i} \in \mathcal{G}, p_{i} \in \mathcal{P}$ and $V\left(x_{i}, p_{i}\right)=0$ for all $i \in \mathbb{N}$, if

$$
\lim _{i \rightarrow \infty} C\left(p_{i}\right)=\infty \quad \Rightarrow \quad \lim _{i \rightarrow \infty} F\left(x_{i}\right)=\infty .
$$

In order to distinguish between complexity specifications and "potential" complexity specifications, we refer to an arbitrary function $C: \mathcal{P} \rightarrow \mathbb{R}_{+}$as a candidate complexity specification. A candidate complexity specification is a complexity specification if it satisfies the conditions given in Definition 2.4.

Property-Proof-Complexity (PPC) specification. The main objective of this paper will be to study all of the aforementioned concepts together as pieces of a whole. This motivates the following definition.

Definition 2.5: A Property-Proof-Complexity specification (or just $P P C$ specification) is a tuple

$$
\left(\mathcal{X}, d_{\mathcal{X}}, \mathcal{G}, \mathcal{P}, \mathcal{R}, V, C\right)
$$

where $\left(\mathcal{X}, d_{\mathcal{X}}, \mathcal{G}\right)$ is a property specification, $(\mathcal{P}, \mathcal{R}, V)$ is a proof specification and $C$ is a complexity specification.

Complexity implies fragility. The motivation for considering PPC specifications lies in their usefulness in formalizing the notion that "complexity implies fragility."

Definition 2.6: Let $\left(\mathcal{X}, d_{\mathcal{X}}, \mathcal{G}, \mathcal{P}, \mathcal{R}, V, C\right)$ be a PPC specification. Complexity implies fragility if for all $(x, p) \in$ $\mathcal{G} \times \mathcal{P}$ such that $V(x, p)=0$,

$$
C(p) \leq F(x) .
$$

\section{COMPLEXITY AND FRAGILITY IN Stability: The Discrete-Time CASE}

In order to illustrate the concepts introduced in the previous section, we will consider the stability of discrete-time dynamical systems of the form:

$$
x_{k+1}=A x_{k},
$$


where $A \in \mathbb{C}^{n \times n}$. In this context, we will introduce a PPC specification and demonstrate how preexisting results, namely [16], can be used to show that complexity implies fragility.

Property specification: discrete-time stability. Since we are considering the stability of discrete-time linear systems, we are interested in the following property specification:

$$
\left(\mathbb{C}^{n \times n}, d_{F}, \mathrm{STDT}\right)
$$

where $d_{F}$ is the metric obtained from the Frobenious norm, \|\|$_{F}$, on $\mathbb{C}^{n \times n}$ and

$$
\operatorname{STDT}=\left\{A \in \mathbb{C}^{n \times n}:\left|\lambda_{i}(A)\right|<1 \text { for } i=1, \ldots, n\right\},
$$

where $\lambda_{i}(A)$ is the $i^{\text {th }}$ eigenvalue of $A$, i.e., STDT is the set of stable discrete-time linear systems. Similarly, let $\mathrm{USTDT}=\mathrm{STDT}^{c}$ be the set of unstable discrete-time linear systems.

Fragility in stability. Given a discrete-time linear system $A$, the fragility of this system is given by:

$$
F(A)=\frac{1}{d_{F}(A, \mathrm{USTDT})} .
$$

The quantity $d_{F}(A, \mathrm{USTDT})$ has been well-studied (cf. [4], [12] and [16]), and is commonly referred to as the distance to instability or the (complex) stability radius. In particular, it can be shown that the distance to instability can be expressed alternatively as follows ${ }^{1}$

$$
d_{F}(A, \mathrm{USTDT})=\min _{\theta \in[0,2 \pi]} \sigma_{\min }\left(e^{j \theta} I-A\right),
$$

which implies that the fragility is given by:

$$
F(A)=\max _{\theta \in[0,2 \pi]} \sigma_{\max }\left(\left(e^{j \theta} I-A\right)^{-1}\right),
$$

It can be easily seen that computing the fragility $\mathrm{F}$ is not intractable, and there are a variety of polynomial time algorithms to do so. On the other hand, the complexity specification that will yield a complexity implies fragility result at the end of this section is a function of the solution to the Lyapunov equation, which is a linear equation and thus easily solved, i.e., the complexity specification can be very easily computed and only uses quantities that are involved in proving that a system is stable. This is indicative of a more general phenomenon that is one of the motivations for complexity implies fragility results-if in the process of proving a property one encounters complexity, it would be beneficial to know that this implies something about the fragility of the system being considered (since distance problems are often intractable).

Proof specification: Lyapunov functions. In the context of discrete-time linear systems, our proofs will consist of quadratic Lyapunov functions, i.e., Lyapunov functions of the form $x^{H} P x$ where $P=P^{H}>0$ is a Hermitian positive

\footnotetext{
${ }^{1}$ We denote the singular values of an $n \times n$ matrix $M$ by $\sigma_{i}(M)$, $i=1, \ldots, n$, the minimum singular value by $\sigma_{\min }(M)$ and the maximum singular value by $\sigma_{\max }(M)$.
}

definite matrix. A proof is verified to be proof of stability of $A$ if it satisfies the discrete-time Lyapunov equation:

$$
P-A^{H} P A=I
$$

That is, to prove stability a Hermitian positive definite matrix $P$ is provided; this is a valid proof if it satisfies the Lyaponov equation.

The notion of Lyapunov functions as proofs can be thus used to define a proof specification of the form:

$$
\left(\mathrm{PD}(n, \mathbb{C}), \mathbb{C}^{n \times n}, \Omega\right),
$$

where $\mathrm{PD}(n, \mathbb{C})$ is the set of $n \times n$ Hermitian positive definite matrices, i.e., the set of proofs, and the verification function $\Omega: \mathbb{C}^{n \times n} \times \operatorname{PD}(n, \mathbb{C}) \rightarrow \mathbb{C}^{n \times n}$ is given by:

$$
\Omega(A, P)=P-A^{H} P A-I .
$$

Remark 3: Proof specifications are not unique as can be seen by considering the discrete-time Lyapunov equation. That is, for every Hermitian positive definition matrix $Q$, there is a proof specification $\left(\operatorname{PD}(n, \mathbb{C}), \mathbb{C}^{n \times n}, \Omega_{Q}\right)$ where $\Omega_{Q}(A, P)=P-A^{H} P A-Q$. We restrict our attention to the proof specification where $Q=I$ for the sake of simplicity, but in some situations it may be desirable to consider families of proof specifications. More generally, picking the "correct" proof specification is an important component of obtaining complexity implies fragility results.

Complexity specification: norm. To complete the PPC specification for discrete-time linear systems with respect to stability, we need to define the complexity of a proof $P \in \mathrm{PD}(n, \mathbb{C})$. There are many possible choices for such a specification, but here we will consider the candidate complexity specification $C: \operatorname{PD}(n, \mathbb{C}) \rightarrow \mathbb{R}_{+}$given by:

$$
C(P):=\sqrt{\|P\|_{2}},
$$

where $\|P\|_{2}=\sigma_{\max }(P)$ is the 2-norm or spectral norm. The following lemma indicates that this is a valid complexity specification in that it satisfies the properties given in Definition 2.4; note that this lemma follows easily from [4].

Lemma 3.1: For the property and proof specifications $\left(\mathbb{C}^{n \times n}, d_{F}, \mathrm{STDT}\right)$ and $\left(\mathrm{PD}(n, \mathbb{C}), \mathbb{C}^{n \times n}, \Omega\right)$, the candidate complexity specification $C: \operatorname{PD}(n, \mathbb{C}) \rightarrow \mathbb{R}_{+}$given in (1) is a complexity specification.

Complexity implies fragility. Combining the formulations introduced in this section, we have thus defined a PPC specification:

$$
\left(\mathbb{C}^{n \times n}, d_{F}, \operatorname{STDT}, \operatorname{PD}(n, \mathbb{C}), \mathbb{C}^{n \times n}, \Omega, C\right) .
$$

Moreover, it has been demonstrated that this specification captures all of the concepts being considered: the systems (discrete-time linear systems), the property (stability), the proof methodology (Lyapunov functions) and the complexity of proof (norm).

Now that a meaningful PPC specification has been defined, the goal is to use this specification to say something useful about the systems being considered. This is done through the 


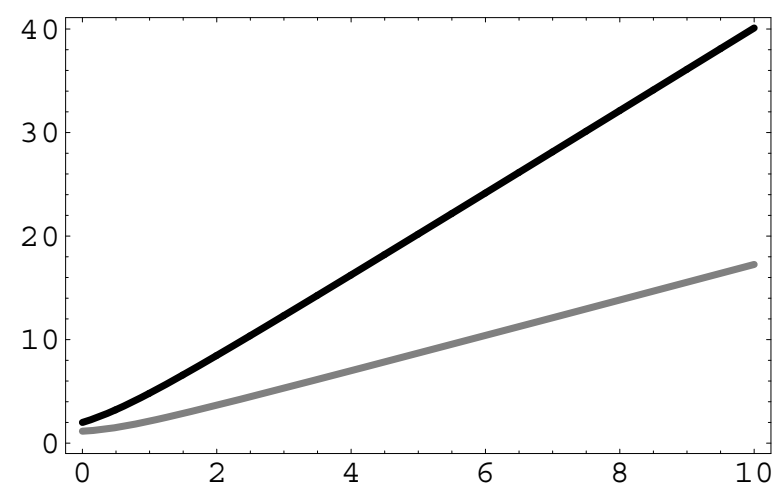

Fig. 1. $C(P(\epsilon))$ (gray) and $F(A(\epsilon))$ (black) versus $\epsilon$.

following complexity implies fragility result (which is just a reformulation of Corollary 4.7 of [16]).

Theorem 1 ([16]): For the PPC specification

$$
\left(\mathbb{C}^{n \times n}, d_{F}, \operatorname{STDT}, \operatorname{PD}(n, \mathbb{C}), \mathbb{C}^{n \times n}, \Omega, C\right)
$$

complexity implies fragility, i.e., for any stable discrete-time linear system $A \in \mathbb{C}^{n \times n}$ and $P \in \operatorname{PD}(n, \mathbb{C})$ such that $\Omega(A, P)=P-A^{H} P A-I=0$,

$$
C(P)=\sqrt{\|P\|_{2}} \leq F(A) .
$$

This theorem demonstrates that meaningful complexity implies fragility results can be obtained. In many ways, this can be viewed as a "canonical" complexity implies fragility result in that it has a clear and precise meaning.

Example 3.1: Consider the discrete-time linear system:

$$
A(\epsilon)=\left(\begin{array}{cc}
\frac{1}{2} & \epsilon \\
0 & \frac{1}{2}
\end{array}\right)
$$

parameterized by $\epsilon \in \mathbb{R}$. Clearly, $A(\epsilon)$ is stable since $\lambda_{1}(A(\epsilon))=\lambda_{2}(A(\epsilon))=1 / 2$ for all $\epsilon$.

Computing $C(P(\epsilon))$, where $P(\epsilon)$ satisfies $P(\epsilon)$ $A^{H}(\epsilon) P(\epsilon) A(\epsilon)=I$, one finds that the proofs of stability become more complex as $\epsilon \rightarrow \infty$, or

$$
\lim _{\epsilon \rightarrow \infty} C(P(\epsilon))=\infty .
$$

Since complexity implies fragility, i.e., $C(P(\epsilon)) \leq F(A(\epsilon))$, this implies that the system $A(\epsilon)$ becomes more fragile as $\epsilon \rightarrow \infty$, or

$$
\lim _{\epsilon \rightarrow \infty} F(A(\epsilon))=\infty .
$$

A graphical representation of this divergent behavior, along with the relative size of $C(P(\epsilon))$ and $F(A(\epsilon))$, can be seen in Figure 1.

\section{Stability of Continuous-Time Linear SYSTEMS: A PPC SPECIFICATION}

In this section, we present a PPC specification related to the stability of continuous-time linear systems, i.e., systems of the form:

$$
\dot{x}=A x
$$

for $A \in \mathbb{F}^{n \times n}$, with $\mathbb{F}=\mathbb{R}$ or $\mathbb{C}$. Since this closely mirrors the PPC specification given in the previous section, we will be brief except where there are notable differences.

Property specification: Continuous-time stability. As with the case of discrete-time systems, since we are interested in the stability of continuous-time systems, consider the following property specification:

$$
\left(\mathbb{F}^{n \times n}, d_{F}, \mathrm{STCT}_{\mathbb{F}}\right)
$$

where $d_{F}$ is the the metric obtained from the Frobenius norm on $\mathbb{F}^{n \times n}$, and

$$
\mathrm{STCT}_{\mathbb{F}}=\left\{A \in \mathbb{F}^{n \times n}: \operatorname{Re}\left(\lambda_{i}(A)\right)<0 \text { for } i=1, \ldots, n\right\},
$$

with $\operatorname{Re}\left(\lambda_{i}(A)\right)$ the real part of the eigenvalue $\lambda_{i}(A)$, is the set of stable continuous-time linear systems. Note that $\mathrm{USTCT}_{\mathbb{F}}=\mathrm{STCT}_{\mathbb{F}}^{c}$ is the set of unstable continuous-time linear systems.

Fragility. Unlike the discrete-time case, we are considering either real or complex linear systems (and thus real or complex perturbations, respectively), i.e., for $A \in \mathrm{STCT}_{\mathbb{F}}$,

$$
F(A)=\frac{1}{d_{F}\left(A, \mathrm{USTCT}_{\mathbb{F}}\right)}
$$

where

$$
d_{F}\left(A, \operatorname{USTCT}_{\mathbb{F}}\right)=\min \left\{\|\Delta A\|_{F}: A+\Delta A \in \operatorname{USTCT}_{\mathbb{F}}\right\},
$$

which is referred to as the real or complex stability radius, depending on whether $\mathbb{F}=\mathbb{R}$ or $\mathbb{C}$, respectively.

The quantity $d_{F}\left(A, \operatorname{USTCT}_{\mathbb{F}}\right)$ was first studied in the seminal paper by Van Loan, [17], in the case when $\mathbb{F}=\mathbb{C}$. It was shown that

$$
d_{F}\left(A, \operatorname{USTCT}_{\mathbb{C}}\right)=\min _{w \in \mathbb{R}} \sigma_{\min }(A-j w I)
$$

This alternate formulation illustrates that computing the complex stability radius is a nontrivial task since $\sigma_{\min }(A-j w I)$ is a nonlinear function, possibly with local minima; this has motivated a wealth of work providing upper and lower bounds on $d_{F}\left(A, \mathrm{USTCT}_{\mathbb{C}}\right)$ (see [5], [7] and [17] to name a few), along with numerical procedures for computing the complex stability radius (cf. [6], [9] and [17]). The real stability radius also has been well-studied, the most notable example of which is [14], where it was shown that

$$
d_{F}\left(A, \operatorname{USTCT}_{\mathbb{R}}\right)=\min _{w \in \mathbb{R}} \max _{\gamma \in(0,1]} \sigma_{2 n-1}\left(\begin{array}{cc}
A & -\gamma w I \\
\frac{w}{\gamma} I & A
\end{array}\right) .
$$

This formula indicates that computing the real stability radius is somewhat difficult but computationally tractable.

Again, when proving stability by solving the Lyapunov equation, we would like to be able to additionally say something useful when this computation becomes hard or ill-conditioned and relate this to the fragility of the system.

Proof specification: Lyapunov functions. As in the case of discrete-time linear systems, our proofs will consist of quadratic Lyapunov functions, $x^{H} P x$ where $P=P^{H}>0$, 
and our proofs will be verified through the continuous-time Lyapunov equation:

$$
A^{H} P+P A=-I .
$$

In particular, consider the following proof specification:

$$
\left(\operatorname{PD}(n, \mathbb{F}), \mathbb{F}^{n \times n}, \Phi\right),
$$

where $\operatorname{PD}(n, \mathbb{F})$ is the set of $n \times n$ Hermitian (or just symmetric when $\mathbb{F}=\mathbb{R}$ ) positive definite matrices, i.e., the set of proofs, and $\Phi: \mathbb{F}^{n \times n} \times \operatorname{PD}(n, \mathbb{F}) \rightarrow \mathbb{F}^{n \times n}$ is given by:

$$
\Phi(A, P)=A^{H} P+P A+I .
$$

Note that if $A$ is stable then there exists a unique $P$ such that $\Phi(A, P)=0$. By slight abuse of notation, we can thus view this $P$ as a function:

$$
\begin{aligned}
P: \mathrm{STCT}_{\mathbb{F}} & \rightarrow \operatorname{PD}(n, \mathbb{F}) \\
A & \mapsto P(A) \text { s.t. } \Phi(A, P(A))=0 .
\end{aligned}
$$

That is, in this case, proofs can be obtained explicitly. This will be clear from context when we view $P$ as a function.

Complexity specification. We now present an admittedly unorthodox notion of complexity for proofs of continuoustime linear systems. In particular, consider the candidate complexity specification $C: \operatorname{PD}(n, \mathbb{F}) \rightarrow \mathbb{R}_{+}$given by:

$$
C(P)=\frac{2}{\left\|P^{-1}\right\|_{F}} .
$$

The following lemma demonstrates that this is a valid complexity specification (see Definition 2.4).

Lemma 4.1: For the property and proof specifications $\left(\mathbb{F}^{n \times n}, d_{F}, \mathrm{STCT}_{\mathbb{F}}\right)$ and $\left(\mathrm{PD}(n, \mathbb{F}), \mathbb{F}^{n \times n}, \Phi\right)$, the candidate complexity specification $C: \operatorname{PD}(n, \mathbb{F}) \rightarrow \mathbb{R}_{+}$given in (3) is a complexity specification.

Proof: Consider any sequence of stable continuoustime linear systems $\left\{A_{i}\right\}_{i=0}^{\infty}, A_{i} \in \mathrm{STCT}_{\mathbb{F}}$, and the corresponding sequence of proofs $\left\{P_{i}\right\}_{i=0}^{\infty}, P_{i} \in \operatorname{PD}(n, \mathbb{F})$, i.e., $\Phi\left(A_{i}, P_{i}\right)=0$ for all $i \in \mathbb{N}$.

Define the following function $K: \mathbb{F}^{n \times n} \rightarrow \mathbb{F}^{n^{2} \times n^{2}}$ given by:

$$
K(A)=A^{T} \otimes I+I \otimes A^{H},
$$

where $\otimes$ is the Kronecker product. It is well-known (cf. [7] and [17]) that $K(A)$ is singular, i.e., $\sigma_{\min }(K(A))=0$ iff there is an eigenvalue of $A$ on the imaginary axis, or

$$
\sigma_{\min }(K(A))=0 \quad \Rightarrow \quad F(A)=\infty .
$$

Now, by (2.17) of [7] (see also [10]), it follows that

$$
\frac{1}{\sqrt{n}}\left\|P_{i}\right\|_{2} \leq\left\|K\left(A_{i}\right)^{-1}\right\|_{2}=\frac{1}{\sigma_{\min }\left(K\left(A_{i}\right)\right)}
$$

for all $i \in N$. Therefore,

$$
\begin{aligned}
\lim _{i \rightarrow \infty}\left\|P_{i}\right\|_{2}=\infty & \Rightarrow \lim _{i \rightarrow \infty} \sqrt{n}\left\|K\left(A_{i}\right)^{-1}\right\|_{2}=\infty \\
& \Rightarrow \lim _{i \rightarrow \infty} \sigma_{\min }\left(K\left(A_{i}\right)\right)=0 \\
& \Rightarrow \lim _{i \rightarrow \infty} F\left(A_{i}\right)=\infty
\end{aligned}
$$

Finally, since $\|M\|_{2} \leq\|M\|_{F}$ for any matrix $M$,

$$
\frac{1}{\left\|P_{i}^{-1}\right\|_{F}} \leq \frac{1}{\left\|P_{i}^{-1}\right\|_{2}}=\sigma_{\min }\left(P_{i}\right) \leq\left\|P_{i}\right\|_{2}
$$

for all $i \in \mathbb{N}$. Therefore,

$$
\begin{aligned}
\lim _{i \rightarrow \infty} \frac{2}{\left\|P_{i}^{-1}\right\|_{F}}=\infty & \Rightarrow \lim _{i \rightarrow \infty}\left\|P_{i}\right\|_{2}=\infty \\
& \Rightarrow \lim _{i \rightarrow \infty} F\left(A_{i}\right)=\infty
\end{aligned}
$$

as desired.

Remark 4: An important consequence of the techniques used to prove Lemma 4.1 is that there is a direct connection between the fragility of $A$, the size of $P$ and the conditioning of the linear system used to compute $P$ :

$$
K(A) \operatorname{Vect}(P)=-\operatorname{Vect}(I),
$$

where Vect is the vector obtained by staking the columns of a matrix. In particular, it was shown in [7] that:

$$
\frac{1}{\sqrt{n}}\|P\|_{2} \leq \frac{1}{\sigma_{\min }(K(A))} \leq \sqrt{n}\|P\|_{2} .
$$

So as $K(A)$ becomes singular, $\|P\|$ becomes large and vice versa. Therefore, if $\|P\|$ is large (or even $C(P)$ given in (3)), it implies the "fragility" (or ill-conditioning) of the linear system (4) along with the fragility of $A$ as will be shown in the next section.

Another complexity specification. We conclude this section by reiterating that complexity specifications are not unique through an example. Consider the candidate complexity specification $\widehat{C}: \operatorname{PD}(n, \mathbb{F}) \rightarrow \mathbb{R}_{+}$given by:

$$
\widehat{C}(P)=\frac{2 \sigma_{\min }(P)}{\sqrt{n}} .
$$

Since $\widehat{C}(P) \leq C(P)$, it follows from Lemma 4.1 that $\widehat{C}(P)$ is a complexity specification.

This illustrates that complexity specifications are not unique and, in fact, there are often whole families of complexity specifications. This indicates that there are some complexity specifications that are "better" than others in that they provide tighter lower bounds for fragility; this will be discussed in further detail following Theorem 3.

\section{Complexity and Fragility in Stability: The Continuous-Time CAse}

In this section, we show that complexity implies fragility for the PPC specification introduced in the previous section, i.e., we show that complexity implies fragility in stability for continuous-time linear systems.

Distance to ill-posedness. Following the approach of [1], we present a technique for bounding the distance to a set of "ill-posed" problems through the use of gradient techniques applied to functions that play the role of "condition numbers." This result will be fundamental in establishing our complexity implies fragility result for continuous-time linear systems. 
Let $X$ be a inner product space, with inner product $\langle$,$\rangle .$ The inner product on $X$ induces a norm $\|\cdot\|=\sqrt{\langle\cdot, \cdot\rangle}$, which induces a metric $d(x, y)=\|x-y\|$. For a smooth function $f: X \rightarrow R$, we denote the Fréchet derivative of $f$ at $x$ by $T_{x} f: X \rightarrow R$, i.e., $T_{x} f(\delta)$ is the derivative of $f$ at $x$ in direction $\delta$. The induced operator norm of $T_{x} f$ is given by:

$$
\left\|T_{x} f\right\|_{\mathrm{op}}=\sup _{\|\delta\|=1}\left|T_{x} f(\delta)\right|,
$$

and the gradient of $f, \nabla f: X \rightarrow X$, is the function uniquely defined as follows:

$$
T_{x} f(\delta)=\langle\nabla f(x), \delta\rangle .
$$

In particular, we have that

$$
\left\|T_{x} f\right\|_{\text {op }}=\|\nabla f(x)\| .
$$

Definition 5.1: Let $X$ be an inner product space, $Y \subset X$, and let $f: Y \rightarrow \mathbb{R}$ be a smooth function. Define $f^{-1}(\infty)$ to be the set such that for every $z \in f^{-1}(\infty)$ there exists a continuous path $x:[0,1) \rightarrow Y$ such that

$$
\lim _{t \rightarrow 1} x(t)=z \quad \text { and } \quad \lim _{t \rightarrow 1} f(x(t))=\infty .
$$

Theorem 2 ([1]): Let $X$ be an inner product space, $Y \subset$ $X$ and let $f: Y \rightarrow \mathbb{R}$ be a smooth function. If for all $x \in Y$ there exists an $M>0$ and $\alpha>1$ such that

$$
\|\nabla f(x)\| \geq M f^{\alpha}(x)
$$

then for all $x \in Y$

$$
d\left(x, f^{-1}(\infty)\right) \leq \frac{1}{(\alpha-1) M f^{\alpha-1}(x)} .
$$

Differentiating the complexity specifiation. Using Theorem 2 as a guide, we will compute the derivative of the complexity specification given in (3) and find a lower bound for the gradient.

Viewing $P$ as a function of $A$ as given in (2), the complexity specification given in (3) can be used to define a function $\kappa: \mathrm{STCT}_{\mathbb{F}} \rightarrow \mathbb{R}_{+}$with:

$$
\kappa(A):=\frac{1}{2} C(P(A))=\frac{1}{\left\|P(A)^{-1}\right\|_{F}} .
$$

We can compute the derivative of $\kappa$ as follows:

Proposition 5.1: For all $A \in \mathrm{STCT}_{\mathbb{F}}$,

$$
T_{A} \kappa(\delta)=\frac{\operatorname{trace}\left(P(A)^{-3} T_{A} P(\delta)\right)}{\left\|P(A)^{-1}\right\|_{F}^{3}},
$$

where $T_{A} P(\delta)$ is the derivative of $P(A)$ which is defined implicitly as follows:

$$
A^{H} T_{A} P(\delta)+T_{A} P(\delta) A=-\left(\delta^{H} P(A)+P(A) \delta\right) .
$$

Proof: We can write

$$
\kappa(A)=\frac{1}{f \circ g \circ P(A)}
$$

where $g(A)=A^{-1}$ and $f(A)=\|A\|_{F}$. The chain and quotient rules yield:

$$
T_{A} \kappa(A)=-\frac{T_{g \circ P(A)} f\left(T_{P(A)} g\left(T_{A} P(\delta)\right)\right)}{\left\|P(A)^{-1}\right\|_{F}^{2}} .
$$

Now the derivatives of $f$ and $g$ can be computed in the standard way, and are given by:

$$
\begin{aligned}
T_{A} g(\delta) & =-A^{-1} \delta A^{-1} \\
T_{A} f(\delta) & =\frac{\operatorname{trace}\left(A^{H} \delta\right)}{\|A\|_{F}}
\end{aligned}
$$

Therefore,

$$
\begin{aligned}
T_{A} \kappa(A) & =\frac{\operatorname{trace}\left(P(A)^{-H} P(A)^{-1} T_{A} P(\delta) P(A)^{-1}\right)}{\left\|P(A)^{-1}\right\|_{F}^{3}} \\
& =\frac{\operatorname{trace}\left(P(A)^{-3} T_{A} P(\delta)\right)}{\left\|P(A)^{-1}\right\|_{F}^{3}}
\end{aligned}
$$

where $P(A)^{-1}=P(A)^{-H}$ since $P(A)$ is Hermitian and positive definite.

Finally, differentiating

$$
\Phi(A, P(A))=A^{H} P(A)+P(A) A+I=0
$$

yields

$$
\delta^{H} P(A)+A^{H} T_{A} P(\delta)+T_{A} P(\delta) A+P(A) \delta=0
$$

as desired.

Using Proposition 5.1, we can establish the following bounds on the gradient of $\kappa$. First, note that $\mathbb{F}^{n \times n}$ is an inner product space with the inner product $\langle A, B\rangle=\operatorname{trace}\left(A^{H} B\right)$; the induced norm is the Frobenius norm.

Proposition 5.2: For all $A \in \mathrm{STCT}_{\mathbb{F}}$,

$$
\|\nabla \kappa(A)\|_{F} \geq 2 \kappa(A)^{2} .
$$

Proof: By (7), it is easy to verify that $T_{A} P\left(P(A)^{-1}\right)=$ $2 P(A)$. Therefore,

$$
\begin{aligned}
\|\nabla \kappa(A)\|_{F} & =\sup _{\delta \neq 0} \frac{\left|T_{A} \kappa(\delta)\right|}{\|\delta\|_{F}} \\
& \geq \frac{\left|T_{A} \kappa\left(P(A)^{-1}\right)\right|}{\left\|P(A)^{-1}\right\|_{F}} \\
& =\frac{\left|\operatorname{trace}\left(P(A)^{-3} T_{A} P\left(P(A)^{-1}\right)\right)\right|}{\left\|P(A)^{-1}\right\|_{F}^{4}} \\
& =2 \frac{\left|\operatorname{trace}\left(P(A)^{-3} P(A)\right)\right|}{\left\|P(A)^{-1}\right\|_{F}^{4}} \\
& =2 \frac{\left|\left\langle P(A)^{-1}, P(A)^{-1}\right\rangle\right|}{\left\|P(A)^{-1}\right\|_{F}^{4}} \\
& =2 \kappa(A)^{2}
\end{aligned}
$$

as desired.

Complexity implies fragility. We now present a novel complexity implies fragility result for the stability of linear systems. This is the main result of this paper.

Theorem 3: For the PPC specification

$$
\left(\mathbb{F}^{n \times n}, d_{F}, \operatorname{STCT}_{\mathbb{F}}, \operatorname{PD}(n, \mathbb{F}), \mathbb{F}^{n \times n}, \Phi, C\right)
$$

complexity implies fragility, i.e., for any stable continuoustime linear system $A \in \mathbb{F}^{n \times n}$ and $P \in \operatorname{PD}(n, \mathbb{F})$ such that $\Phi(A, P)=A^{H} P+P A+I=0$,

$$
C(P)=\frac{2}{\left\|P^{-1}\right\|_{F}} \leq F(A) .
$$


Proof: By Theorem 2 and Proposition 5.2, we know that for all $A \in \mathrm{STCT}_{\mathbb{F}}$

$$
d\left(A, \kappa^{-1}(\infty)\right) \leq \frac{1}{2 \kappa(A)}=\frac{1}{C(P(A))}=\frac{\left\|P(A)^{-1}\right\|_{F}}{2} .
$$

The fact that $C$ is a complexity specification implies that $\kappa^{-1}(\infty) \subset \mathrm{USTCT}_{\mathbb{F}}$. This in turn implies that $d\left(A, \mathrm{USTCT}_{\mathbb{F}}\right) \leq d\left(A, \kappa^{-1}(\infty)\right)$.

Another complexity implies fragility result. Just as complexity specifications are not unique, complexity implies fragility results are not unique. To provide an example of this, consider the PPC specification:

$$
\left(\mathbb{F}^{n \times n}, d_{F}, \operatorname{STCT}_{F}, \operatorname{PD}(n, \mathbb{F}), \mathbb{F}^{n \times n}, \Phi, \widehat{C}\right),
$$

where $\widehat{C}$ is given in (5). Then for this PPC complexity implies fragility, or for all $A \in \mathrm{STCT}_{\mathbb{F}}$ and $P \in \mathrm{PD}(n, \mathbb{F})$ such that $\Phi(A, P)=0$,

$$
\widehat{C}(P)=\frac{2 \sigma_{\min }(P)}{\sqrt{n}} \leq F(A) .
$$

Of course, this is just a consequence of Theorem 3 and the fact that $\widehat{C}(P) \leq C(P)$, but it illustrates that complexity implies fragility results are far from unique and motivates the following observations on this theorem.

Comments on Theorem 3. It has been indicated numerous times that complexity specifications are not unique to a given problem and proof specification. This implies that there are complexity specifications, or complexity implies fragility results, that are "better" than others. In fact, the authors believe that the complexity implies fragility result presented in Theorem 3 is far from the best one in that it will fail to detect fragility in a variety of situations (as Example 5.2 will illustrate).

In an ideal situation, a complexity specification would always be small exactly because the problems being considered are not fragile-most situations do not display this ideal property, i.e., the complexity is not always small exactly because problems can be fragile. Given the fact that complexity is unavoidable, and due to the non-uniqueness of complexity specifications, it is natural to ask: "what is the 'best' complexity specification?" Intuitively the best complexity specification would be the one that provides the tightest lower bound on the fragility and/or always detects fragile systems, i.e., a complexity specification where fragility also implies complexity. More specifically, for the PPC specification given in Theorem 3, it was shown in [17] (see also [5] and [10]) that when $\mathbb{F}=\mathbb{C}$ and for $A \in \mathbb{R}$,

$$
F(A) \leq 2\|P\|_{2} .
$$

Therefore, an "ideal" complexity implies fragility result would be the one that utilizes the complexity specification $C(P)=c\|P\|_{2}$ for some constant $c \in \mathbb{R}_{+}$; this will be further justified in the next paragraph. In [17], Van Loan discusses the possibility of such a complexity implies fragility result (although he does not use this language) but admits to not being able to prove such a result and gives a good explanation of why such a proof is difficult. We have, similarly, been unable to prove such a result. Therefore, establishing whether such a complexity implies fragility result exists is a very interesting open problem.

Other interpratations of complexity and fragility. In the previous paragraph, we discussed complexity specifications related to $\|P\|$. It is important to note that $P$ relates to other important system-theoretic concepts (we will discuss these in the context of continuous-time systems, but analogous considerations hold for discrete-time systems). We will briefly mention two of these: transient response and $\mathcal{H}_{\infty}$ control.

First note that $P$ directly relates to the transient response of $A$ (see [7]). More specifically, if $x(t)$ is the solution to $\dot{x}=A x$ with $x(0)=x_{0}$, then

$$
\left\|x_{0}\right\|^{2} \sigma_{\min }(P) \leq x_{0}^{H} P x_{0}=\int_{0}^{\infty}\|x(t)\|^{2} d t \leq\left\|x_{0}\right\|^{2}\|P\| .
$$

So the area under a solution, which can be viewed as a way of detecting fragility in stability in the system, directly relates to both the minimum and maximum singular value of $P$, i.e., $P$ contains more information about $A$ than just a yes/no answer on stability.

Another interesting insight into complexity and fragility for linear systems is provided by $\mathcal{H}_{\infty}$ control (cf. [3] and [18]). We first note that the transfer function for the system:

$$
\begin{aligned}
\dot{x} & =A x+u \\
y & =x
\end{aligned}
$$

is given by $G(s)=(s I-A)^{-1}$. Therefore, the $\mathcal{H}_{\infty}$ norm of this transfer function is:

$$
\|G(s)\|_{\infty}=\max _{w \in \mathbb{R}}\left\|(j w I-A)^{-1}\right\|=F(A),
$$

or the $\mathcal{H}_{\infty}$ norm is just the fragility of $A$. Moreover, $\|G(s)\|_{\infty}<\gamma$ if and only if there exists an $X(\gamma)=$ $X(\gamma)^{H}>0$ satisfying the algebraic Riccati equation:

$$
A^{H} X(\gamma)+X(\gamma) A+\frac{1}{\gamma^{2}} X(\gamma)^{2}=-I
$$

It follows that $\lim _{\gamma \rightarrow \infty} X(\gamma)=P$. That is, as the system becomes more fragile, which forces $\gamma$ to get large, $X(\gamma)$ converges to $P$. Thus, the $\mathcal{H}_{\infty}$ norm, fragility in stability of a linear system, Riccati equations and Lyapunov equations all relate. These relationships have yet to yield any new complexity implies fragility results, but it seems likely that the continued study of these interconnections will yield interesting insights.

Examples. We conclude this section with a couple of examples illustrating the results presented. In all of these examples, we take $\mathbb{F}=\mathbb{C}$ as it is generally easier to compute the complex stability radius $d\left(A, \mathrm{USTCT}_{\mathbb{C}}\right)$.

Example 5.1: The first example will demonstrate that in some "ideal" cases, the notion of complexity introduced is directly related to fragility.

Consider the continuous-time linear system

$$
A(\epsilon)=\left(\begin{array}{cc}
-\epsilon & 1 \\
-1 & -\epsilon
\end{array}\right)
$$




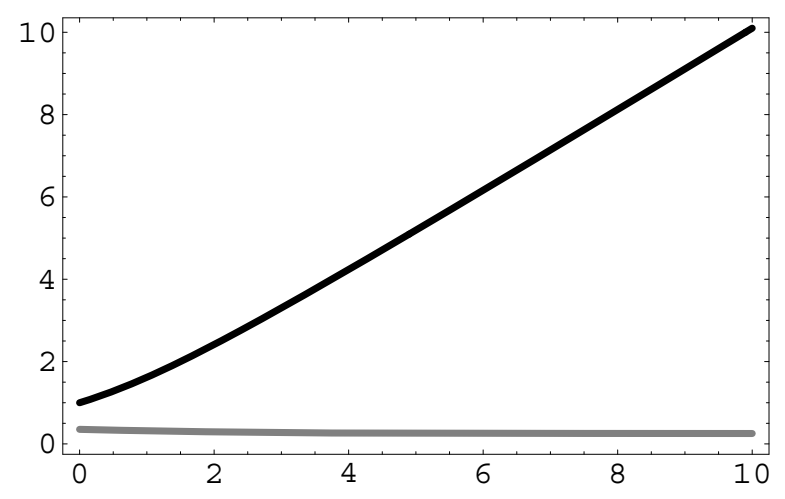

Fig. 2. $\quad C(P(\epsilon))$ (gray) and $F(A(\epsilon))$ (black) versus $\epsilon$.

with $\epsilon \in \mathbb{R}_{+}$. Since $\lambda_{1}(A(\epsilon))=-\epsilon+j$ and $\lambda_{2}(A(\epsilon))=$ $-\epsilon-j, A(\epsilon)$ is stable for all $\epsilon>0$ and the eigenvalues approach the imaginary axis as $\epsilon \rightarrow 0$.

For this example, it is easy to verify that

$$
C(P(\epsilon))=\frac{1}{\epsilon \sqrt{2}} \leq F(A(\epsilon))=\frac{1}{\epsilon} .
$$

Therefore, $C(P(\epsilon)) \rightarrow \infty$ as $\epsilon \rightarrow 0$ implying that the system becomes fragile as $\epsilon \rightarrow 0$.

Example 5.2: Unlike the previous example, this example will demonstrate that the notion of complexity introduced can fail to notice certain fragile systems.

In analogy to Example 3.1, consider the continuous-time linear system:

$$
A(\epsilon)=\left(\begin{array}{cc}
-1 & \epsilon \\
0 & -1
\end{array}\right)
$$

with $\epsilon \in \mathbb{R}$. Clearly $A(\epsilon)$ is stable for all $\epsilon \in \mathbb{R}$ since $\lambda_{1}(A(\epsilon))=\lambda_{2}(A(\epsilon))=-1$.

For this example, $C(P(\epsilon))$ does not diverge as $\epsilon \rightarrow \infty$ while $F(A(\epsilon)) \rightarrow \infty$ (see Figure 2). That is, the complexity specification fails to detect the fact that the problem is becoming fragile. This indicates that there are "better" complexity specifications as discussed in the comments on Theorem 3.

\section{CONCLUSIONS}

This paper presented an axiomatic formulation of complexity implies fragility through the use of property specifications, proof specifications and complexity specifications, or PPC specifications. These concepts were illustrated in the context of stability for both discrete-time and continuoustime systems. This work is only meant to be the first step in both formalizing the notion of complexity implies fragility and providing examples of this phenomenon. There are clearly "better" complexity implies fragility results related to the stability of continuous-time systems, and there are many other complexity implies fragility results that could be established in the context of systems theory, e.g., results related to stabilizability, controllability and nonlinear stability.

The greatest challenge in obtaining far-reaching complexity implies fragility results is to better understand the many forms that these results can take. In this paper we picked a notion of fragility that is natural, and we connected it to the complexity of proofs. The authors believe that although it probably is universally true that proof complexity implies some type of fragility, the notions of complexity and fragility may be different, i.e., a complex proof may imply a type of fragility different than the one considered in this paper. Therefore, given a proof method, a promising research direction is improving notions of complexity so that they better reflects the real failure in algorithms and improving notions of fragility so that they better reflects fragilities that are natural and meaningful.

\section{ACKNOWLEDGEMENT}

The authors are grateful to Maryam Fazel for the many discussions on complexity and fragility.

\section{REFERENCES}

[1] J. W. Demmel, "On condition numbers and the distance to the nearest ill-posed problem," Numerische Mathematik, vol. 51, pp. 251-289, 1987.

[2] A. Dickman, "On the robustness of multivariable linear feedback systems in state-space representation," IEEE Transactions on Automatic Control, vol. 32, no. 5, pp. 407-410, 1987.

[3] J. C. Doyle, K. Glover, P. P. Khargonekar, and B. A. Francis, "Statespace solutions to standard $\mathcal{H}_{2}$ and $\mathcal{H}_{\infty}$ control problems," IEEE Transactions on Automatic Control, vol. 34, no. 8, pp. 831-847, 1989.

[4] P. M. Gahinet, A. J. Laub, C. S. Kenney, and G. A. Hewer, "Sensitivity of the stable discrete-time lyapunov equation," IEEE Transactions on Automatic Control, vol. 35, no. 11, pp. 1209-1217, 1990.

[5] C. He, "On the distance to uncontrollability and the distance to instability and their relation to some condition numbers in control," Numerische Mathematik, vol. 76, pp. 463-477, 1997.

[6] C. He and G. A. Watson, "An algorithm for computing the distance to instability," SIAM Journal of Matrix Analysis and Applications, vol. 20, no. 1, pp. 101-116, 1998.

[7] G. Hewer and C. Kenney, "The sensitivity of the stable lyapunov equation," SIAM Journal of Control and Optimization, vol. 26, pp. 321-244, 1988

[8] N. J. Higham, "Matrix nearness problems and applications," in Applications of Matrix Theory, M. J. C. Gover and S. Barnett, Eds. Oxford University Press, 1989, pp. 1-27.

[9] N. J. Higham, M. Konstantinov, V. Mehrmann, and P. Petkov, "The sensitivity of computational control problems," IEEE Control Systems Magazine, pp. 28-43, February 2004.

[10] C. Kenney and G. Hewer, "The sensitivity of the algebraic and differential riccati equations," SIAM Journal of Control and Optimization, vol. 28, pp. 50-69, 1990 .

[11] M. Mesbahi, "Robustness analysis via the running time of the interior point methods," Systems and Control Letters, vol. 44, no. 5, pp. 355$361,2001$.

[12] T. Mori, "On the relationship between the sectral radius and the stability radius for discrete systems," IEEE Transactions on Automatic Control, vol. 35, no. 7, p. 835, 1990.

[13] P. A. Parrilo, "Structured semidefinite programs and semialgebraic geometry methods in robustness and optimization," Ph.D. dissertation, California Institute of Technology, 2000

[14] L. Qiu, B. Bernhardsson, A. Rantzer, E. J. Davison, P. M. Young, and J. C. Doyle, "A formula for the computation of the real stability radius," Automatica, vol. 31, no. 6, pp. 879-890, 1995.

[15] J. Renagar, "Some perturbation theory for linear programming," in Mathematical Programming. Springer, 1994, vol. 65, pp. 73-91.

[16] M. Tippett, S. Cohn, R. Todling, and D. Marchesin, "Conditioning of the stable discrete-time lyapunov operator," SIAM Journal of Matrix Analysis and Applications, vol. 22, pp. 56-65, 2000.

[17] C. F. Van Loan, "How near is a stable matrix to an unstable matrix?" in Linear Algebra and its role in systems theory, ser. Conntemporary Mathematics. AMS, 1985, vol. 47, pp. 465-478.

[18] K. Zhou, J. C. Doyle, and K. Glover, Robust and Optimal Control. Prentice Hall, 1995. 